\title{
LARGE-SCALE MAGNETIC STRUCTURES RESPONSIBLE FOR CORONAL DISTURBANCES
}

\author{
V. BUMBA \\ Astronomical Institute of the Czechoslovak Academy of Sciences, Ondřejov Observatory, Czechoslovakia \\ and \\ J. SÝK OR A \\ Astronomical Institute of the Slovak Academy of Sciences, Skalnaté Pleso Observatory, Czechoslovakia
}

\begin{abstract}
In the first part of our communication, in a short summary of our recent results, it is demonstrated that over the last 15 years (the time interval for which the magnetic synoptic charts are available) the largest solar activity, usually connected with proton-flare occurrence, has been very closely related to a characteristic large-scale pattern in the magnetic field distribution with a life-time of the order of 8-10 solar rotations. These regular features are seen in the negative as well as in the positive polarity, although they can be seen better in the negative polarity where their forms are more pronounced, regardless of the activity cycle. The form alternates with the location in the northern or the southern solar hemispheres due to the mutual relations of both polarities, individual active regions, the influence of the differential rotations, the shift in longitude, etc. This pattern could be observed up to the large August 1972 proton-flare activity.

The large-scale magnetic patterns described are accompanied by characteristic large-scale features in the green $(\lambda 5303)$ coronal emission, presented in the form of isophotes on the synoptic charts (reduced to a unified photometric scale).

In the second part of the presentation, preliminary results, concerning the correlation of the longitudinal distribution of the green coronal emission with the negative and the positive polarity fields for two time intervals (August 1960-September 1961 and January 1969-December 1969) are described. The existence of two 'coronal active longitudes' in both intervals, as well as the close relation of these longitudinal emission maxima to certain parts of the large-scale characteristic bodies of negative polarity, is discussed. Also, the existence of one heliographic longitude, connected with 'coronal holes' (minimal green corona emission), and its relation to the positive polarity large-scale pattern are proved.
\end{abstract}

\section{Introduction}

The greatest coronal disturbances are connected with large solar flares, above all with particle-emitting flares. The active regions in which these flares occur, have not only a specific magnetic field configuration, but this magnetic situation seems to develope within the frame of a slowly changing large-scale distribution of the background magnetic field patterns in the solar atmosphere. Using the solar magnetic field synoptic charts, constructed from the Mt. Wilson Observatory daily disk maps, we have tried several times to study the regularities and recurrences in this distribution and the dynamics of its development, especially in relation to the distribution of solar activity, and of the green corona and large solar flares (see, for example, Bumba and Howard, 1969; Ambrož et al., 1971; Bumba, 1972a, b; Bumba and Sýkora, 1973). In the present note we would, first of all, like to give a short summary of our results as concerns the close relationship of particle emitting flares and a certain regular type of large-scale magnetic field distribution, which is reflected in the distribution of green corona emission, over the last fifteen years. Using the com- 
parison of the longitudinal distribution of the green coronal emission with that of the magnetic fields, we are trying to find some physical meaning for the striking morphological regularities.

\section{Particle-Emitting Flares and the Regular Patterns of the Large-Scale Distribution of Solar Magnetic Fields and Green Corona}

Two years ago, it was demonstrated that during the declining part of the last 19th cycle of solar activity, the flares which are followed by a particle emission, observed as cosmic ray or PCA events, tend to correlate with a specific pattern of the background magnetic field (Bumba, 1972a). Of 46 flares, accompanied by type IV radiobursts, observed between August 1959 and September 1963 in 22 active regions, about 32 , or possibly 40 flares in all or possibly 17 active regions occurred in this characteristic magnetic field configuration. (4 flares in 3 active regions were not correlated because of the poor quality of the synoptic maps.) Although the number of such flares is also relatively large during the present 20th cycle, this problem has not yet been treated statistically, because it will still take years to complete the list of flares. But several of the largest events have been examined with regard to this behaviour, and the same relation to practically the same form of magnetic features has been found, including the August 1972 large active region (Bumba et al., 1972; Bumba and Sýkora, 1972a, b; Bumba, 1973).

The best example of the complex situation in the background magnetic field distribution connected, with a proton-flare region, may be seen displayed in both polarities in colour and in the purest form on the synoptic chart for rotation No. 1428 in our Atlas (Howard et al., 1967), where the mean heliographic position of the centre is about $130^{\circ}$ in longitude and about $12^{\circ} \mathrm{N}$ in latitude. The magnetic field pattern may be described even better on synoptic charts where the polarities are drawn separately (Figures 1, 2 and 3). The events in negative polarity are related to a drop-shaped (we call it 'supergiant') structure with its tail in the higher latitudes of the northern hemisphere. The drop with its head to the west, formed from older activity regions, more stable and regular, is usually about $90^{\circ}-100^{\circ}$ long. The centre of gravity of the major solar activity and of the particle-emitting flare occurrence is located at the eastern part of the feature, where the activity changes more rapidly, just below the root of the tail. The area of positive polarity forms a 'mirror' image to that of the negative polarity with its tail stretched out to the southern latitudes and with its body fitting into the drop of the negative polarity feature.

The development of the described magnetic patterns is a very complex process, because morphologically both magnetic polarities studied separately on a large scale do not develop simultaneously and in phase with regard to time and heliographic longitude. The life-time of a 'supergiant' structure is of the order of one year although it can best be observed only for a few rotations.

Correlating the synoptic charts of the green ( $\lambda 5303)$ coronal emission distribution, drawn in the form of isophotes from coronal data which were reduced to a unified 

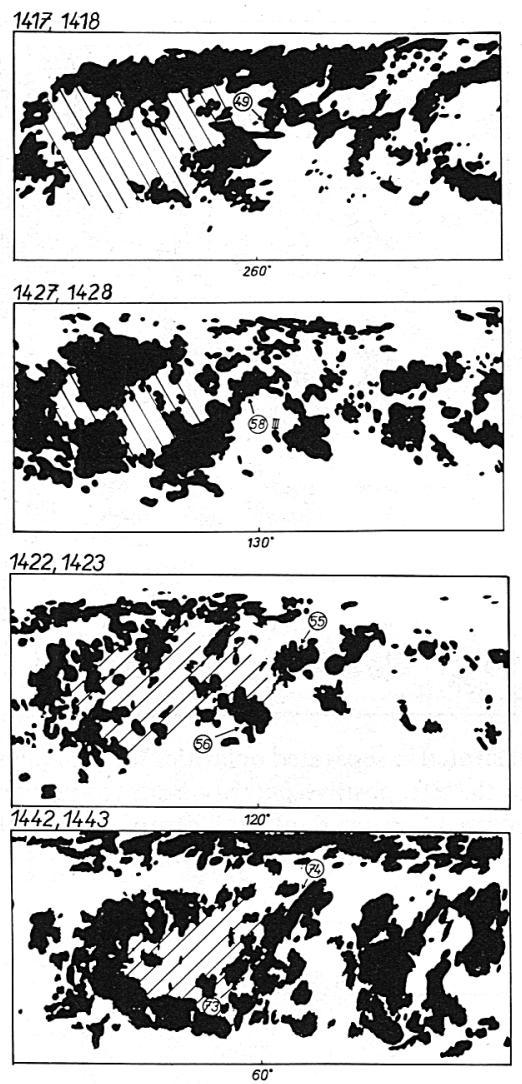
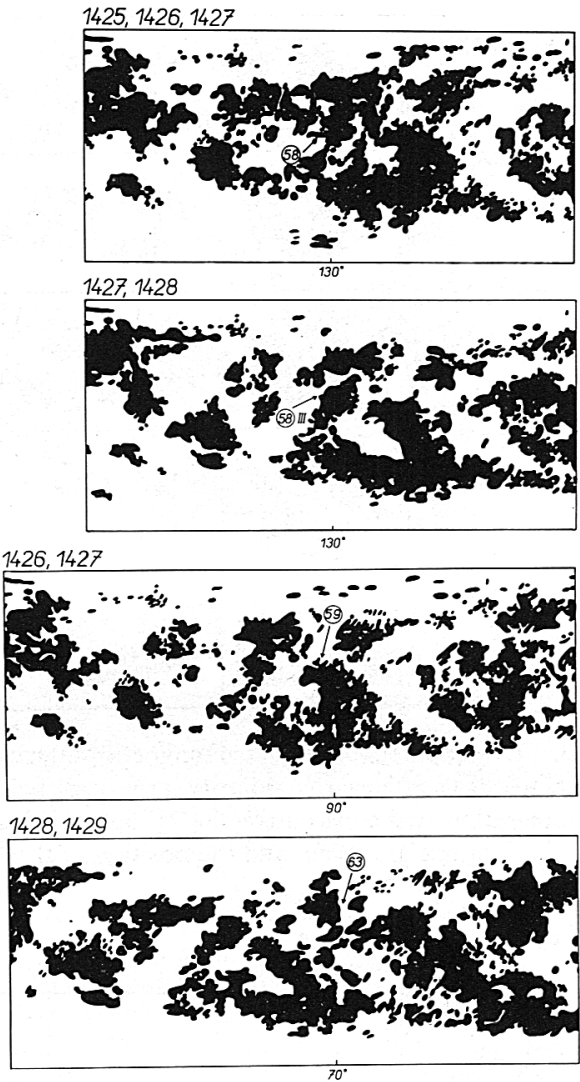

Fig. 1. Examples of the drop-shaped ('supergiant') structures to which the occurrence of particle-emitting flares is related in negative (to the left) and positive (to the right) polarity large-scale magnetic fields. The numbers of rotations of the magnetic charts, two of which were overlapped for integration, are shown, as well as the heliographic longitude of the centre of the magnetic map in the neighbourhood of which the flare centre is located (indicated by an arrow and a number in a circle). The tails, located at higher latitudes of the northern hemisphere for the negative polarity and of the southern hemisphere for the positive polarity bodies, can be seen.

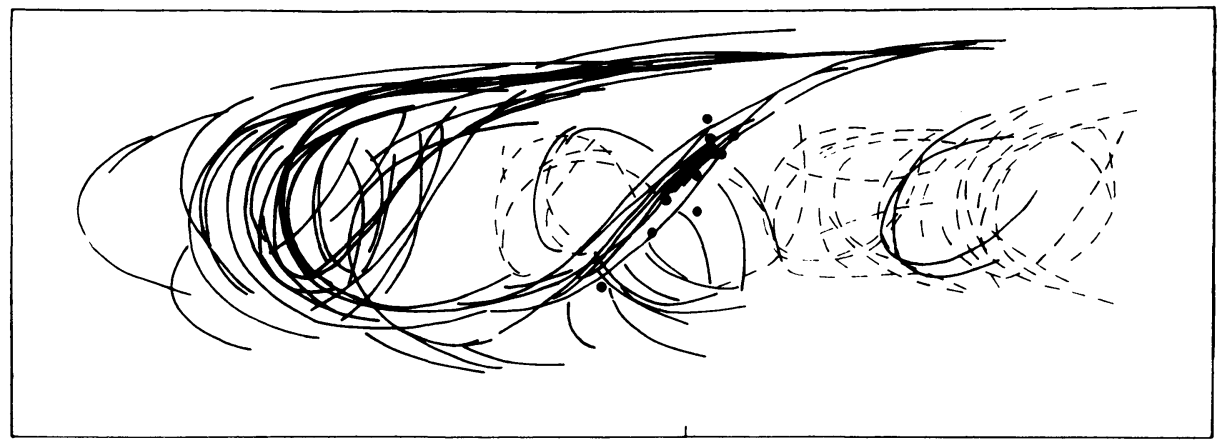

Fig. 2. Diagram of overlapping of best visible drop-shaped ('supergiant') negative polarity structures to which the particle-emitting flares with their positions, indicated by full circles, are related. 

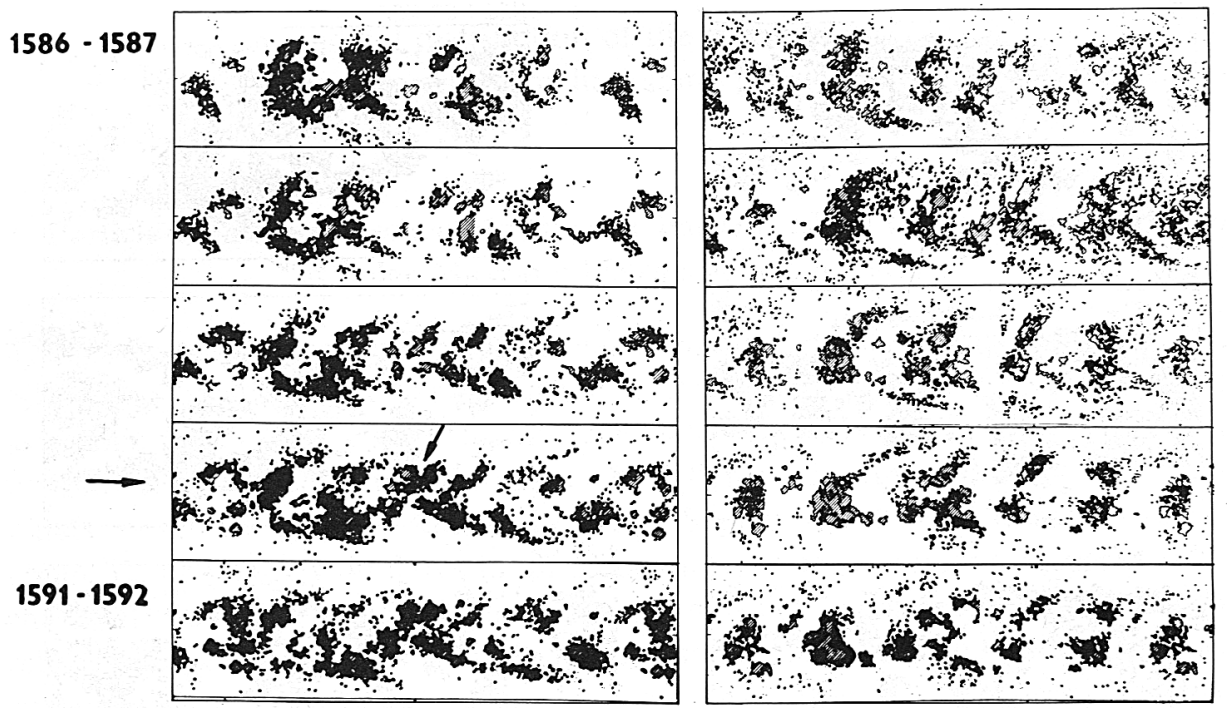

Fig. 3. Development of discussed magnetic patterns, demonstrated in separated polarities for the August 1972 active region. Negative polarity large-scale fields are to the left, positive polarity fields to the right. For integration two consecutive charts, one of which is repeated, are overlapped. The numbers of the rotations are given, and the position of the August active region is indicated by arrows.

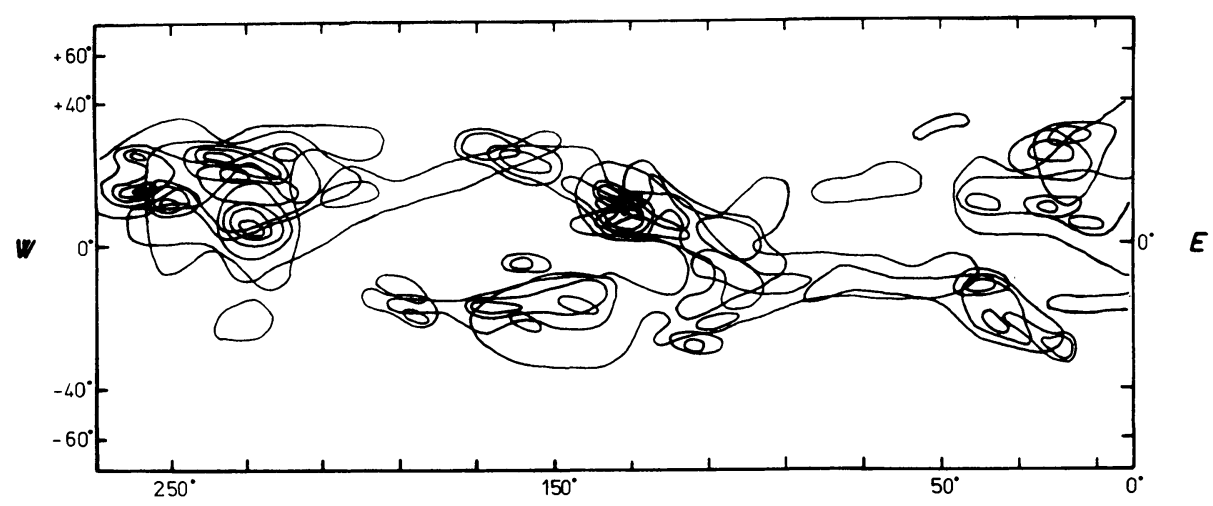

Fig. 4. Example of the 'supergiant' structure to which the occurrence of particle-emitting flares is related (positions of flares are indicated by full circles) in the distribution of the green coronal emission (only the maximal isophotes were drawn). Three synoptic charts (Nos. 1425, 1426 and 1427) were overlapped.

photometric scale (Sýkora, 1971, 1972) with the same large flare active regions, we may see that big elliptical features, formed from an enhanced coronal emission, conform practically with the 'supergiant' body of the magnetic field. The emission is concentrated to the periphery of this body and is usually higher above the protonflare region (Figures 4 and 5). 


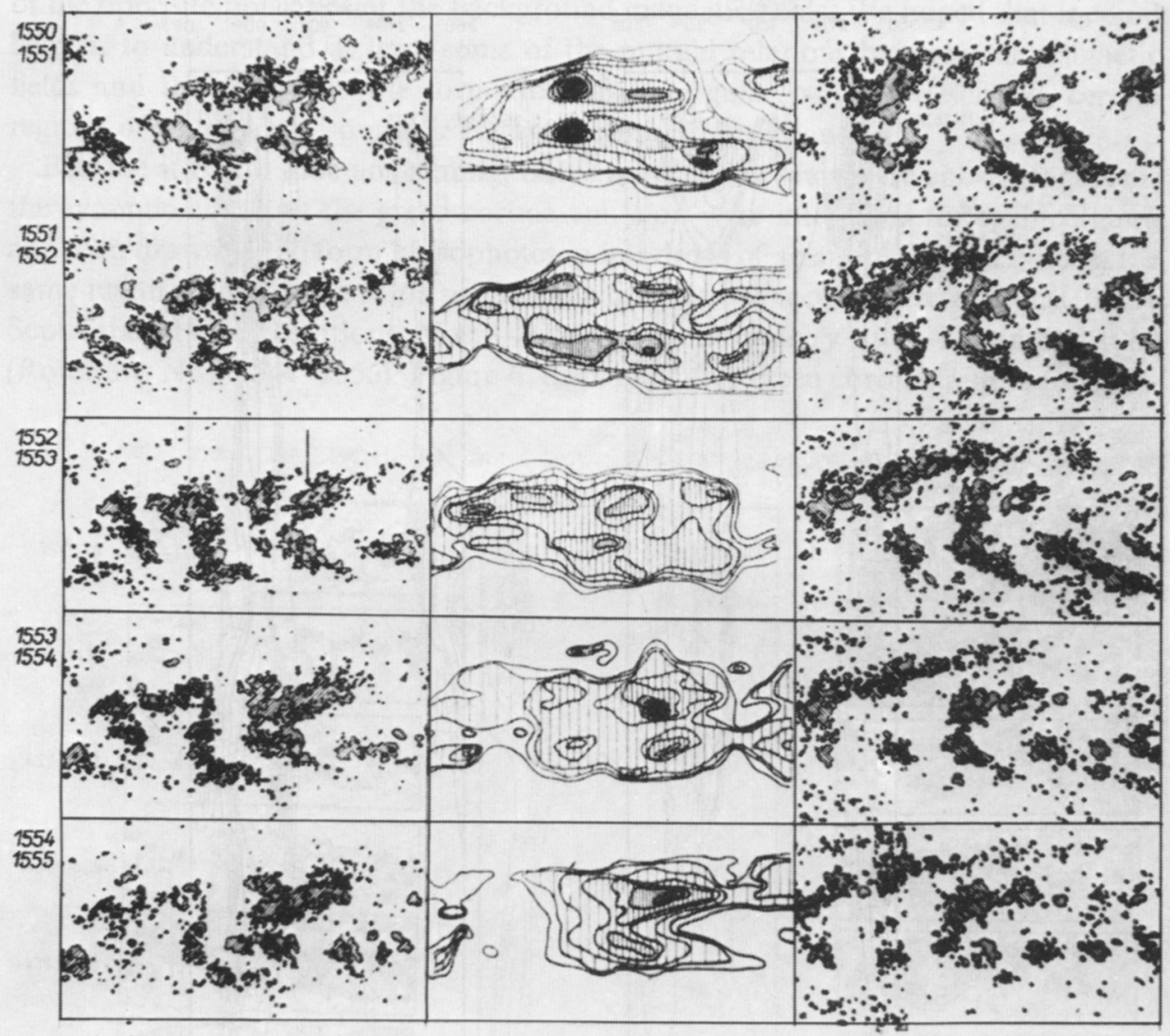

Fig. 5. Parts of synoptic charts (Nos. 1530-1555) of the distribution of the green coronal emission (in the middle part of the figure) and of the same magnetic synoptic charts, drawn in separated polarities (positive on the left side, negative on the right side of the picture) for the proton-flare event of November 2, 1969. For integration two consecutive magnetic maps, one of which is repeated, are overlapped. The development of the large complex magnetic pattern, connected with the evolution of green coronal characteristic feature, may be observed. The position of the particle-emitting flare is visualized by an arrow.

\section{Longitudinal Distribution of the Green Coronal Emission and its Relation to the Background Magnetic Fields}

Several attempts have already been made to show how the 'supergiant' regular structures of the background magnetic fields develope from regularities in the longitudinal distribution of these fields (see, for example, Ambrož et al., 1971; Bumba, 1972a). It is a very complicated problem, connected with the question of the reality of the so-called 'active longitudes' and their reasons.

It seemed to us, therefore, that it may be of some interest to study the longitudinal distribution of the green coronal emission and its relation to the same distribution 


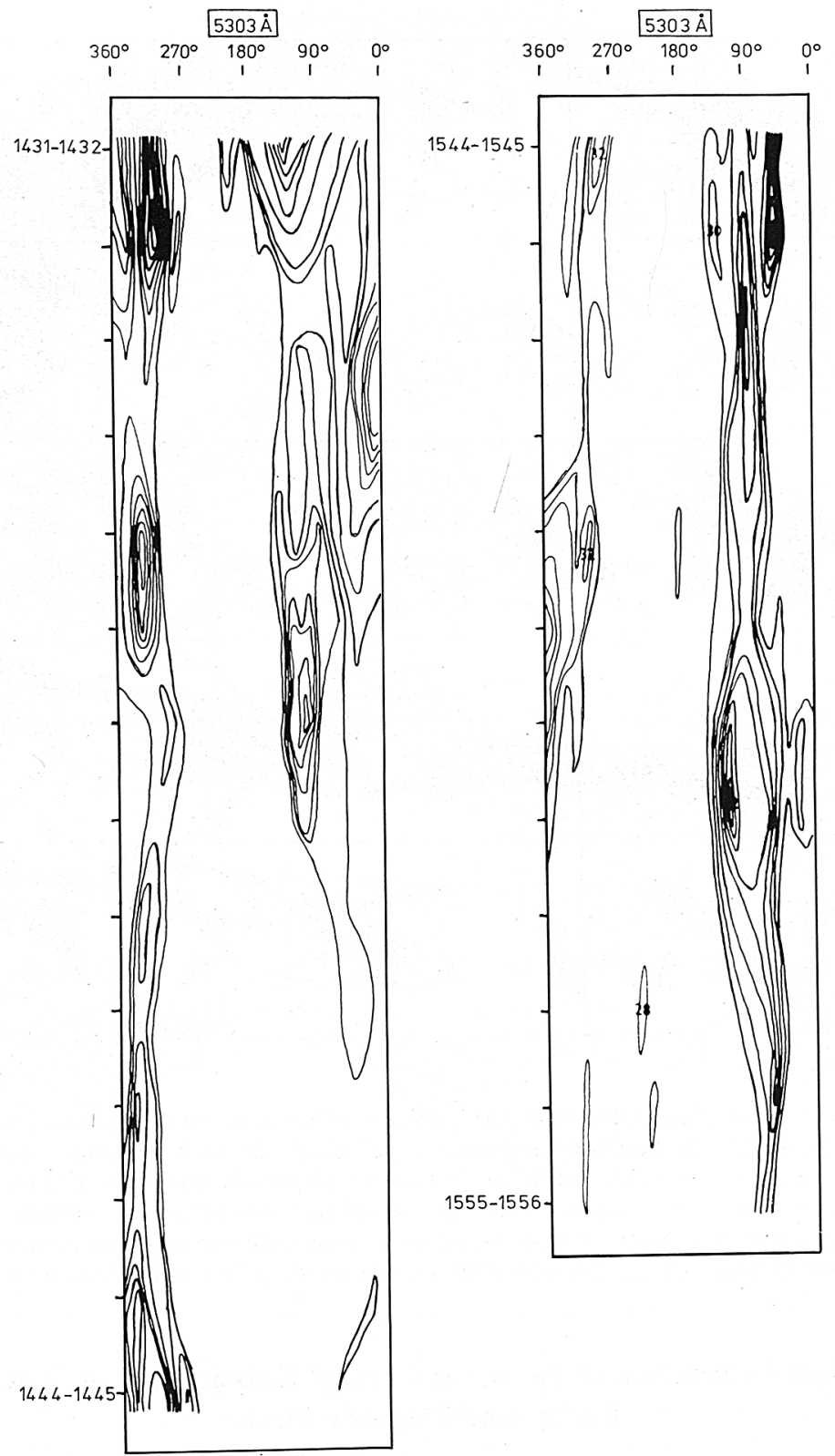

Fig. 6. The longitudinal distribution of the green ( $\lambda 5303)$ coronal emission, demonstrated by the isophotes in hundreds of absolute coronal units for two investigated time intervals: August 1960-September 1961 (Rotations Nos. 1431-1444) to the left and to the right January 1969-December 1969 (Rotations Nos. 1544-1556). For integration two consecutive rotations are overlapped. The heliographic longitudes are indicated at the top of each picture. 
of the opposite polarities of the background magnetic fields. We hoped that it could help us to understand at least some of the mutual relations between the magnetic fields and the corona in the formation of the regular patterns described, certain regions of which are responsible for large coronal disturbances.

Because it is still time consuming, only two time intervals were chosen for them; the synoptic charts of the green corona emission were integrated for each rotation and then drawn in the form of isophotes in hundreds of absolute coronal units in the same manner and on the same scale as the magnetic synoptic charts: August 1960September 1961 (Rotations Nos. 1431-1444) and January 1969-December 1969 (Rotations Nos. 1544-1556). Figure 6, displaying this green corona longitudinal dis-

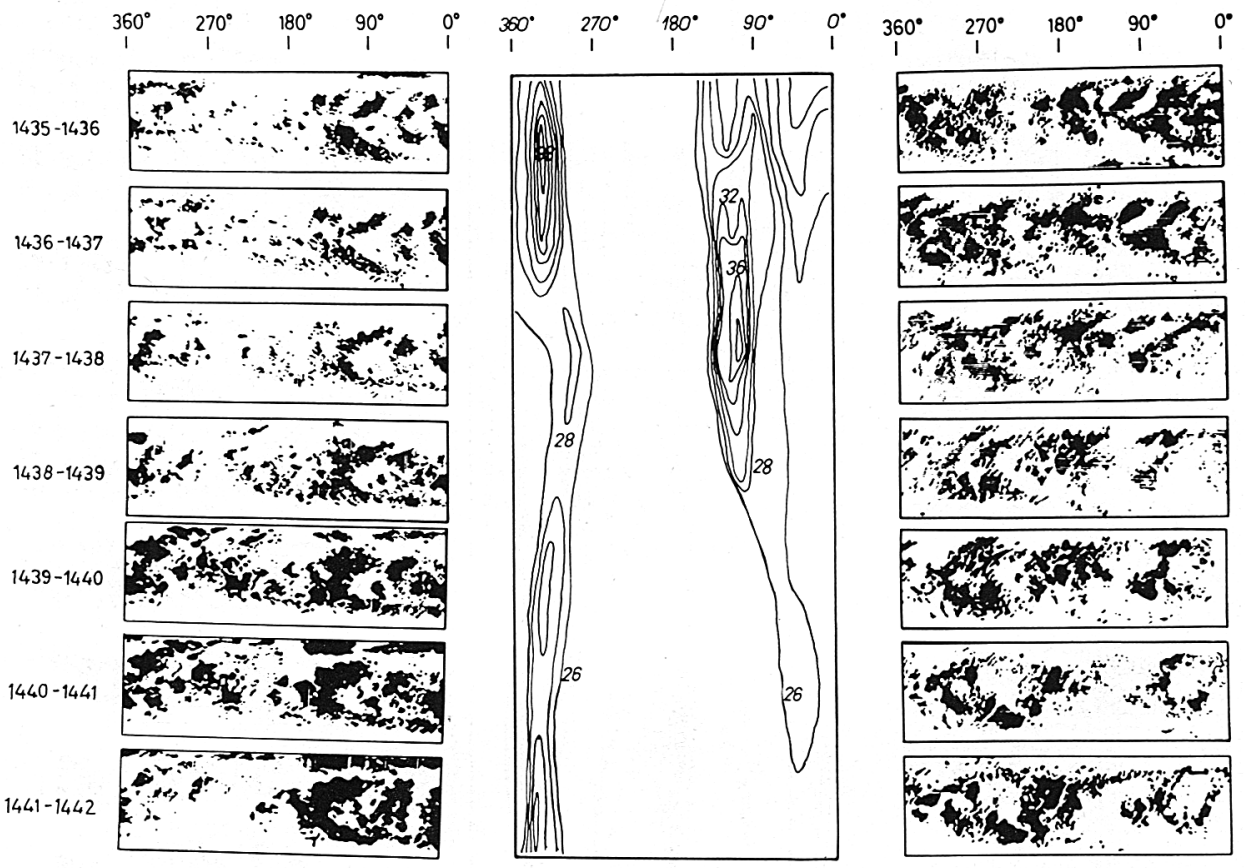

Fig. 7. The longitudinal distribution of green ( $\lambda$ 5303) coronal emission for rotations Nos. 1435-1442 demonstrated by isophotes in hundreds of absolute coronal units drawn in the same manner as the magnetic synoptic maps shown on each side of the coronal data. Magnetic synoptic charts are constructed in separated polarities (negative to the left, positive to the right). For integration two consecutive maps, one of which is repeated, are overlapped. The heliographic longitudes are indicated at the top of each picture.

tribution, demonstrates that during both periods the green coronal emission had two maxima in the heliographic longitude: one around $90^{\circ}-120^{\circ}$ and the second one around $320^{\circ}-340^{\circ}$. This means that they are again not symmetrically spaced in heliographic longitude like many other solar phenomena. The sudden shifts in position of this maximal coronal emission, amounting to several tens of degrees in the longitude seemed to be striking. The internal structure of the individual emission maxima - their changes in time - not only seem to be connected with the evolution of activity, 


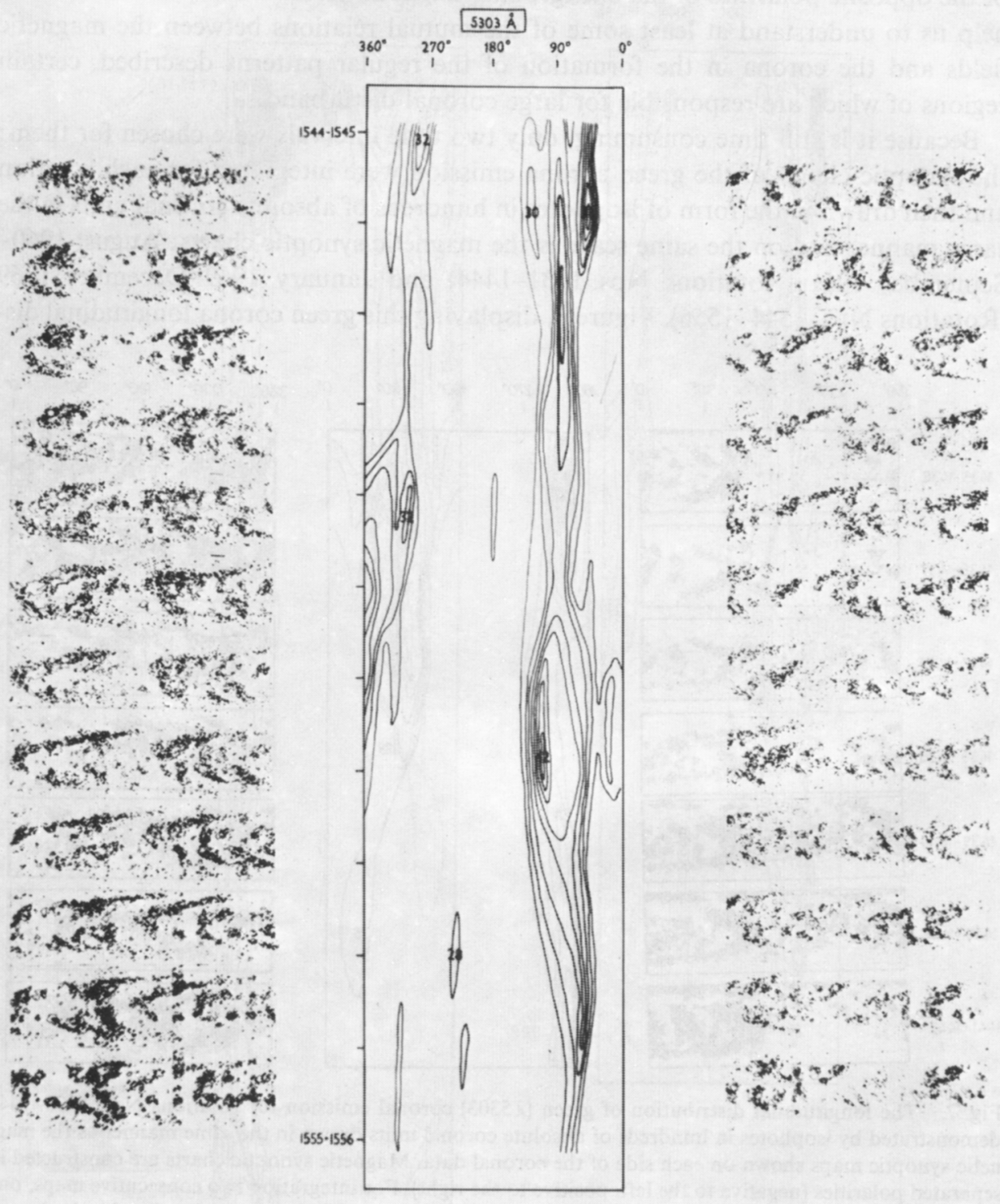

Fig. 8. The longitudinal distribution of the green $(\lambda 5303)$ coronal emission for rotations Nos. 1544-1556 demonstrated by isophotes in hundreds of absolute coronal units drawn in the same manner as the magnetic synoptic maps shown on each side of the coronal data. Magnetic synoptic charts are constructed in separated polarities (negative to the left, positive to the right). For integration two consecutive maps, one of which is repeated, are overlapped. The heliographic longitudes are indicated at the top of the middle picture. 
but also with different rotational periods of the active regions in various latitudes, etc. This problem certainly has to be studied separately for the whole time interval, for which the basic observational material for the corona (Sýkora, 1972) is available.

It has already been demonstrated (Ambrož et al., 1971; Bumba, 1972a, b; Bumba and Sýkora, 1973) that the large-scale magnetic features of negative polarity tend to be closely related, at least during the declining phase of the recent cycle and during the increasing phase of the present cycle of solar activity, to the regions with developing solar activity and the positive polarity features, formed from older fields, to the regions which seem to be the source of the quiet solar wind. The same facts seem to be reflected in the correlation of the green coronal emission maxima with the background magnetic field patterns. During the first time interval, the maxima of the coronal emission coincide well in position with the negative polarity features (Figure 7). When a 'supergiant' structure is fully developed, the position of the coronal maximum agrees with the eastern part of this structure, i.e. with the region the large flares tend to be connected with. Even the longitudinal shift of the coronal maximum

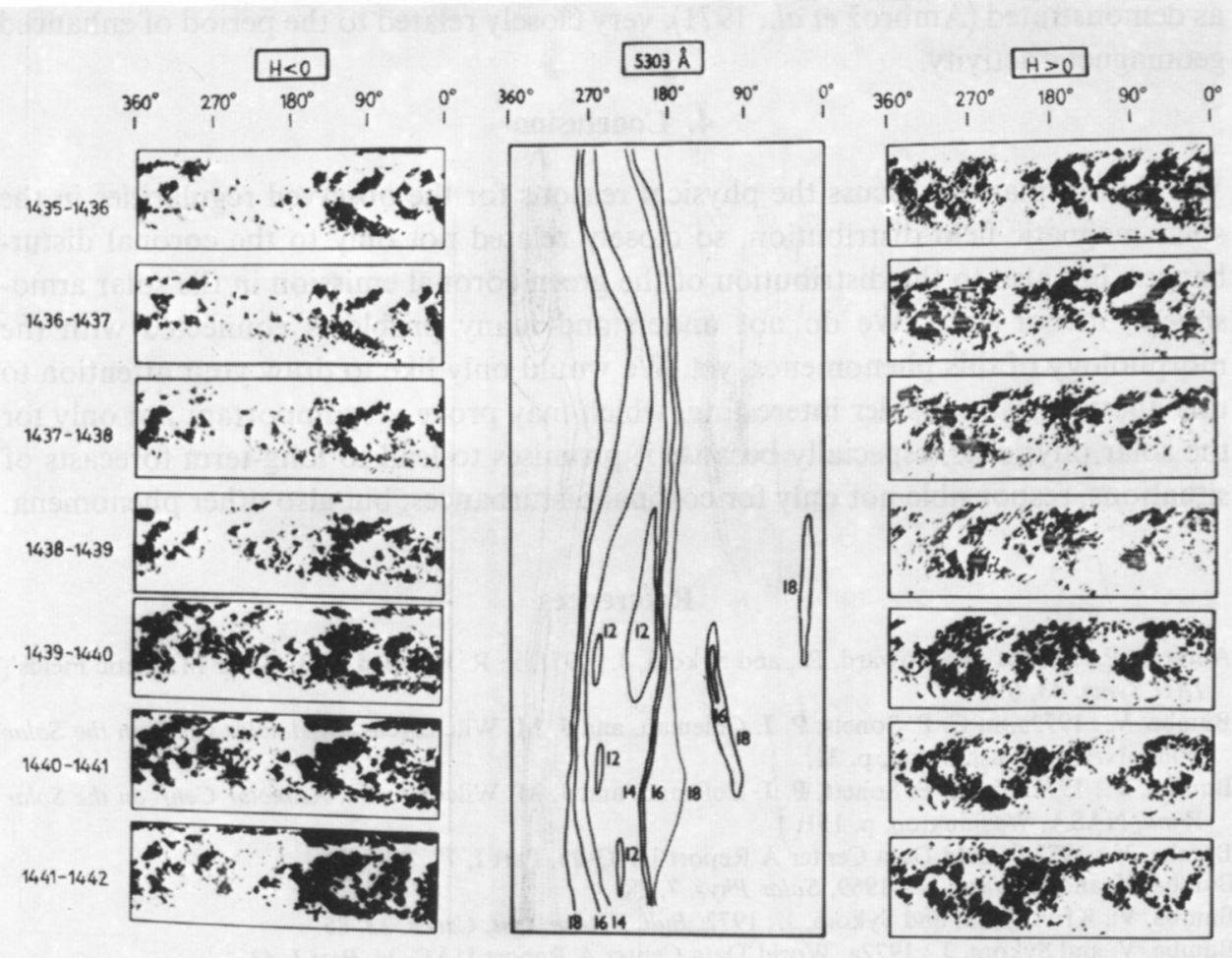

Fig. 9. The isophotes of the minimal green coronal emission in hundreds of absolute coronal units for rotations Nos. 1435-1442, drawn in the same manner as the magnetic synoptic charts, shown on each side of the coronal data: negative polarity maps to the left and positive polarity charts to the right. Again two consecutive rotations are overlapped for integration and the heliographic longitudes are indicated at the top of each picture. 
demonstrates the shift of solar activity from the head of such a structure to the root of its tail. As concerns the correlation with positive polarity patterns, it again agrees with what was said above. But we have to underline that the 'mirror positive polarity inclusion' of the negative 'supergiant' body does not agree with the large positive regular 'supergiant' structure, which developed, for example, during the studied time interval in the position $\left(\sim 200^{\circ}\right)$ opposite to that of the negative polarity 'supergiant' structure. The same seems to be true of the second time interval (Figure 8), although the magnetic situation there is more complicated. During both time intervals protonflare regions were observed in agreement with our results.

Just recently 'holes' in the corona have been discussed frequently. We succeeded in constructing the isophotes of the minimum green coronal emission only for our first studied time interval. Comparing the position of the minimum coronal emission, in Figure 9, in heliographic longitude with the synoptic charts of magnetic field, drawn in separate polarities, we may see that it coincides well with the areas minimally occupied by negative polarity fields, on the one hand, but, on the other, it agrees very well with the position of the positive 'supergiant body' which seems to be, as demonstrated (Ambrož et al., 1971), very closely related to the period of enhanced geomagnetic activity.

\section{Conclusion}

We did not plan to discuss the physical reasons for the observed regularities in the solar magnetic field distribution, so closely related not only to the coronal disturbances, but also to the distribution of the green coronal emission in the solar atmosphere, in our note. We do not understand many problems connected with the morphology of this phenomenon yet. We would only like to draw your attention to this question, we consider interesting, which may prove very important not only for the solar physicists, especially because it promises to lead to long-term forecasts of situations, responsible not only for coronal disturbances, but also other phenomena.

\section{References}

Ambrož, P., Bumba, V., Howard, R., and Sýkora, J.: 1971, in R. Howard (ed.), 'Solar Magnetic Fields', IAU Symp. 43, 696.

Bumba, V.: 1972a, in C. P. Sonett, P. J. Coleman, and J. M. Wilcox (eds.), Asimolar Conf. on the Solar Wind, NASA, Washington, p. 31.

Bumba, V.: 1972b, in C. P. Sonett, P. J. Coleman, and J. M. Wilcox (eds.), Asimolar Conf. on the Solar Wind, NASA, Washington, p. 151.

Bumba, V.: 1973, World Data Center A Report UAG-28, Part I, 77.

Bumba, V. and Howard, R.: 1969, Solar Phys. 7, 28.

Bumba, V., Křivský, L., and Sýkora, J.: 1972, Bull. Astron. Inst. Czech. 23, 85.

Bumba, V. and Sýkora, J.: 1972a, World Data Center A Report UAG-24, Part I, 43.

Bumba, V. and Sýkora, J.: 1972b, World Data Center A Report UAG-24, Part II, 311.

Bumba, V. and Sýkora, J.: 1973, Space Research XIII, Akademie-Verlag, Berlin, d 23-1.

Howard, R., Bumba, V., and Smith, S.: 1967, Carnegie Inst. of Washington Publ. No. 626, Washington, D.C.

Sýkora, J. : 1971, Bull. Astron. Inst. Czech. 22, 12.

Sýkora, J. : 1972, Contr. Astron. Obs. Skalnaté Pleso 5, in press. 


\section{DISCUSSION}

Mullaly: I would like to draw attention to a 'gap' in the positions of microwave bursts (1958-61) as reported by Krishnan and Mullaly.

Boischot: Why does the differential rotation not show in your synoptic maps?

Bumba: Differential rotation appears with individual active regions but the large scale magnetic pattern indicates rigid rotation.

Stix: The hydromagnetic equations governing the solar dynamo have, besides the axisymmetric solutions describing the 22 year cycle, also $\phi$-dependent solutions. Such solutions may provide a theoretical explanation of the supergiant structures mentioned by Dr Bumba. They have the interesting property that their sector boundaries do not change their shape as they slowly drift in longitude. 